\title{
Modal Experiment on Structural Condition Monitoring Using Cumulative Harmonic Analysis
}

\author{
Yoshinori Takahashi, ${ }^{1}$ Satoru Goto, ${ }^{2}$ and Mikio Tohyama ${ }^{2}$ \\ ${ }^{1}$ Tokyo Metropolitan College of Industrial Technology, 8-17-1 Minami Senju, Arakawa-ku, Tokyo 116-0003, Japan \\ ${ }^{2}$ Graduate School of Fundamental Science and Engineering, Waseda University, 3-4-1 Okubo Shinjyuku-ku, Tokyo 1698555, Japan
}

Correspondence should be addressed to Yoshinori Takahashi, yoshinori@ieee.org

Received 3 July 2011; Accepted 23 November 2011

Academic Editor: Luc Gaudiller

Copyright ( 2012 Yoshinori Takahashi et al. This is an open access article distributed under the Creative Commons Attribution License, which permits unrestricted use, distribution, and reproduction in any medium, provided the original work is properly cited.

\begin{abstract}
This paper describes a cumulative harmonic analysis ( $\mathrm{CHA}$ ) that replaces the stepwise time window function of Berman and Fincham's cumulative spectral analysis with a spectral accumulation function, thereby enabling a new structural health monitoring method. CHA estimates and visualizes system damping conditions without the need of transient-vibration records. The damping conditions are closely related to the spectral distribution around the dominant spectral peaks due to structural resonance. This type of spectral distribution can be visualized with CHA even within a short interval of random vibration samples. The effect of $\mathrm{CHA}$ on monitoring the damping conditions was reported by the authors in a previous study. In the current study, the authors confirmed the usefulness of CHA for structural condition monitoring by conducting scale-model experiments.
\end{abstract}

\section{Introduction}

This study aims at visualizing the spectral changes in random structural vibrations due to external noise. Spectral changes in a vibration might be informative for monitoring the health of a system and for structural diagnostics. In one of the standard methods, a test signal is given to the subject and the transmitted vibration at the other point is observed [1]. Abnormalities in the health of a structure are detectable from the time variance of the damping constant seen in the transfer function. However, the health of a structure can only be evaluated with a general measurement method that requires a test signal. Instead, if the changes in harmonic characteristics could be analyzed from the subtleties of structural vibrations caused by external noise, the health of the structure would be able to be monitored at any time. However, it is difficult to track random variations by using the structural transfer functions independent of the source signal characteristics. This is because the transfer function analysis of structural vibration systems generally requires the external source characteristics to be specified $[2,3]$.

Hirata proposed a method for monitoring invisible changes in a structure on the basis of the frequency distributions of the dominant spectral components during short-interval periods (SIPs) under random and nonstationary vibration conditions without placing assumptions or restrictions on the specific source signal [4]. The maximum point of the cumulative curves of the SIP distribution are used to estimate the resonant frequency, and the sharpness of the distribution can be evaluated from the statistical frequency of the dominant spectral components. As the variance in the distribution increases, the modal bandwidth widens, thus increasing the damping. Recently, Hirata and coworkers conducted experiments on health monitoring of a modal structure using its nonstationary vibrations, wherein SIP distributions were used for detecting changes in the dynamic properties of a structure [5]. The maximum points of the SIP distribution without the magnitude were used to prevent bad influences from the source variation and from the resonant frequency.

Different from Hirata's work, the authors proposed a new spectral analysis method using a cumulative harmonic analysis (CHA) that includes growing a resonant spectral peak through spectral accumulation of a long interval [6]. A very short frame length is used for SIPs, on the order of the one-twentieth of the target vibration period. The 

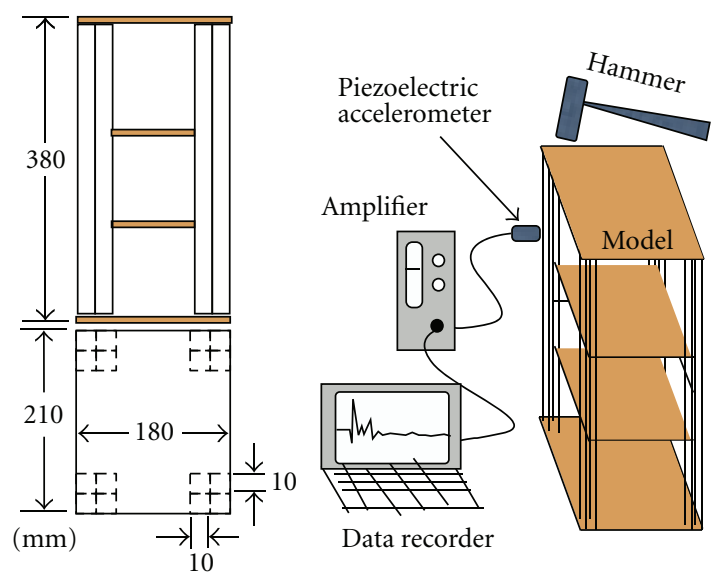

FIgURE 1: Scale model and experimental system.
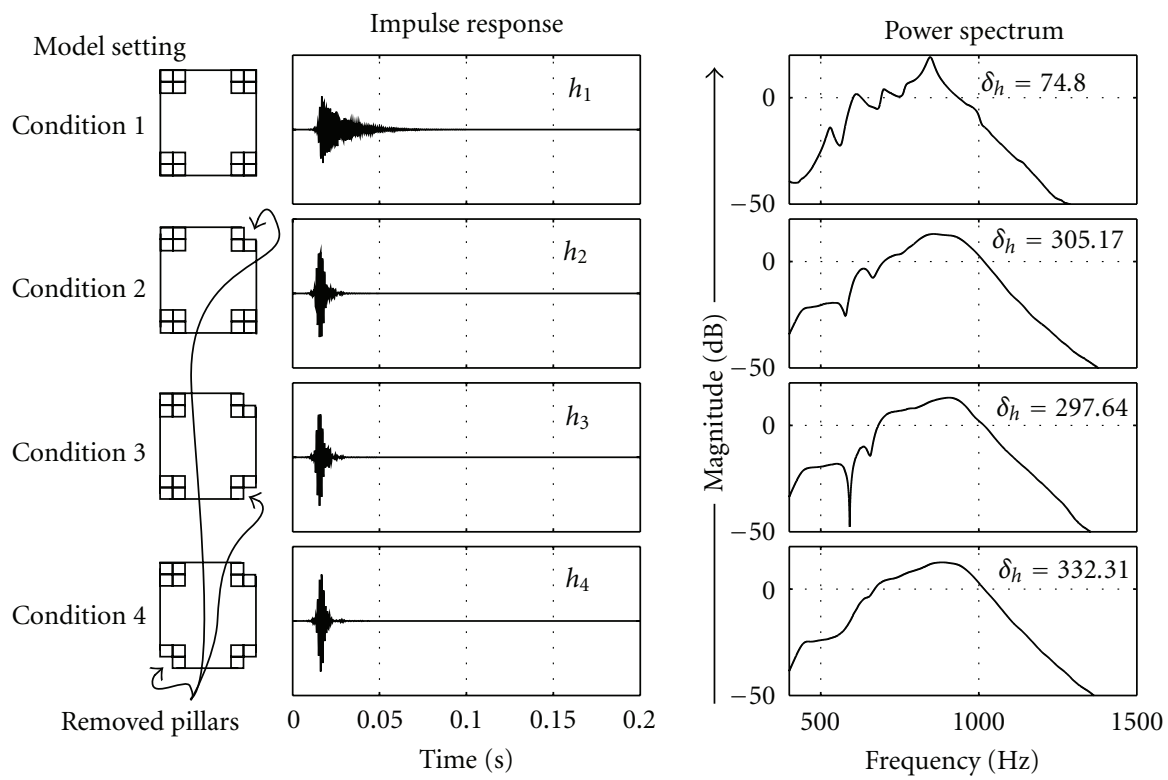

FIGURE 2: Measured responses and conditions.

SIP distribution is obtained from several thousand frames. However, the frame length of CHA uses a few hundred target vibration periods, and an accumulation window is used to produce effects similar to the SIP distribution. The spectralaccumulation process of CHA emphasizes the spectral peak of a hidden resonance, such as the transfer function in a short-framed signal. It does not, however, emphasize the resonance peaks of a fluctuating frequency. This means that CHA is good for visualizing the resonant information of the transfer function from a short frame of a random source vibration. We already discussed how to visualize damping changes from a random structural vibration signal by using the CHA spectral variance and presented computer simulation results [7].

In this paper, we discuss the frame length and efficiency of diagnostics using a kind of nonstationary source. We also describe experiments on a scale model that were conducted to determine CHA's ability to find damping changes from environmental vibrations. Through these investigations and experiments, we show that CHA is a good way of monitoring the modal bandwidth changes in transfer functions when the external source is unknown.

In Section 2, we describe the scale model for experiment and the impulse response measurement. In Section 3, we explain the analysis methods, CHA, normal short-time Fourier transform (STFT), and exponential windowing (EW), which is a general analytical method for diagnostics. We discuss the results of the experiment in Section 4.

\section{Scale Model and the Impulse Response}

It is difficult to make a structural model which produces the required damping change for a short time. The impulse 


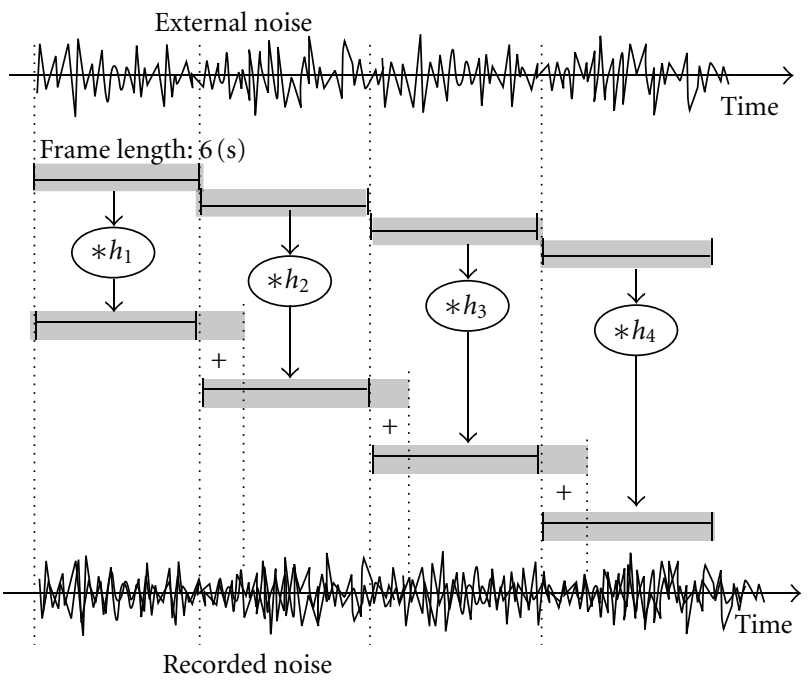

FIgURE 3: Time variant convolution.

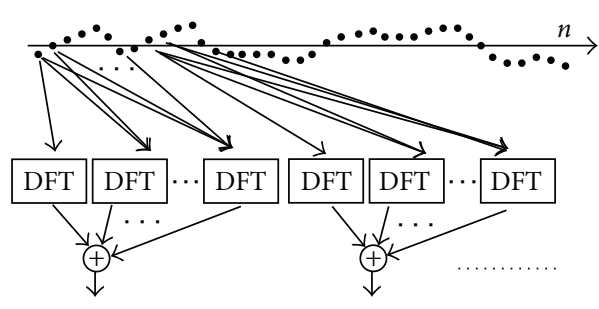

(a)

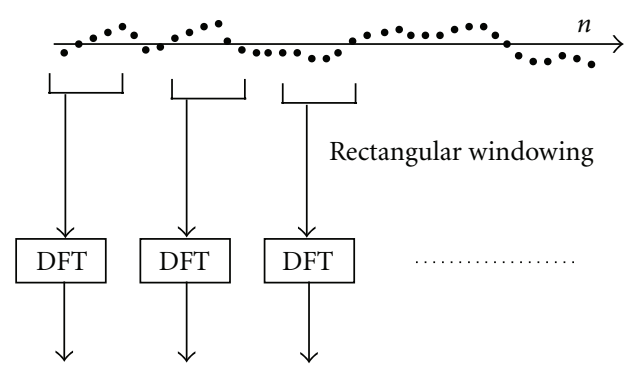

(c)

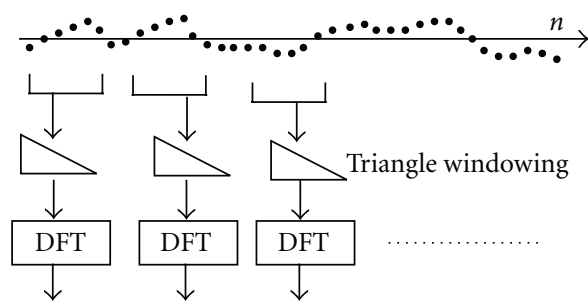

(b)

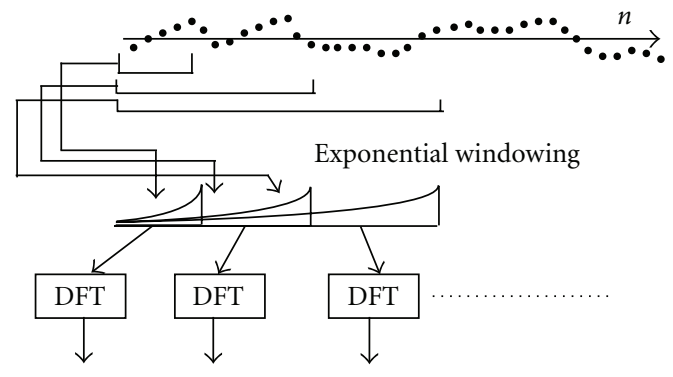

(d)

Figure 4: Analysis methods. (a) CHA, (b) CHA with triangular windowing, (c) STFT (short-time Fourier transform), and (d) EW (exponential windowing).

responses of a scale model were recorded (Figure 1). The model had three floors with four groups of wooden pillars. The response to an impulsive hammer was recorded with a piezoelectric accelerometer (PV-90B, RION). The measurements were carried out for different responses obtained under different conditions (the number of pillars was varied). Figure 2 shows the measured response waveforms with the power spectral records for each measurement condition. A band-pass Butterworth filter of the 6th order and a passband of $600 \mathrm{~Hz}<f_{\text {pass }}<1000 \mathrm{~Hz}$ was used in the analysis.

The external noise was assumed to be unknown. Four types of noise were used in the numerical experiments: white noise, recorded noise from an aircraft, street noise, and sea waves recorded at a beach. Different impulse responses, measured under different conditions, were convolved with the external noise every 6 seconds (Figure 3 ).

\section{Experimental Analysis Methods}

Figure 4(a) illustrates the CHA method. One defines the function

$$
\operatorname{CHA}\left(n, \mathrm{e}^{-j \Omega}\right) \equiv \sum_{m=0}^{n} w(m) x(m) \mathrm{e}^{-j \Omega m}
$$

of the framed input signal $x(n),(0 \leq n<N)$. The spectralaccumulation function $w(m)$ is

$$
w(m) \equiv n-m+1, \quad(0 \leq m \leq n) .
$$




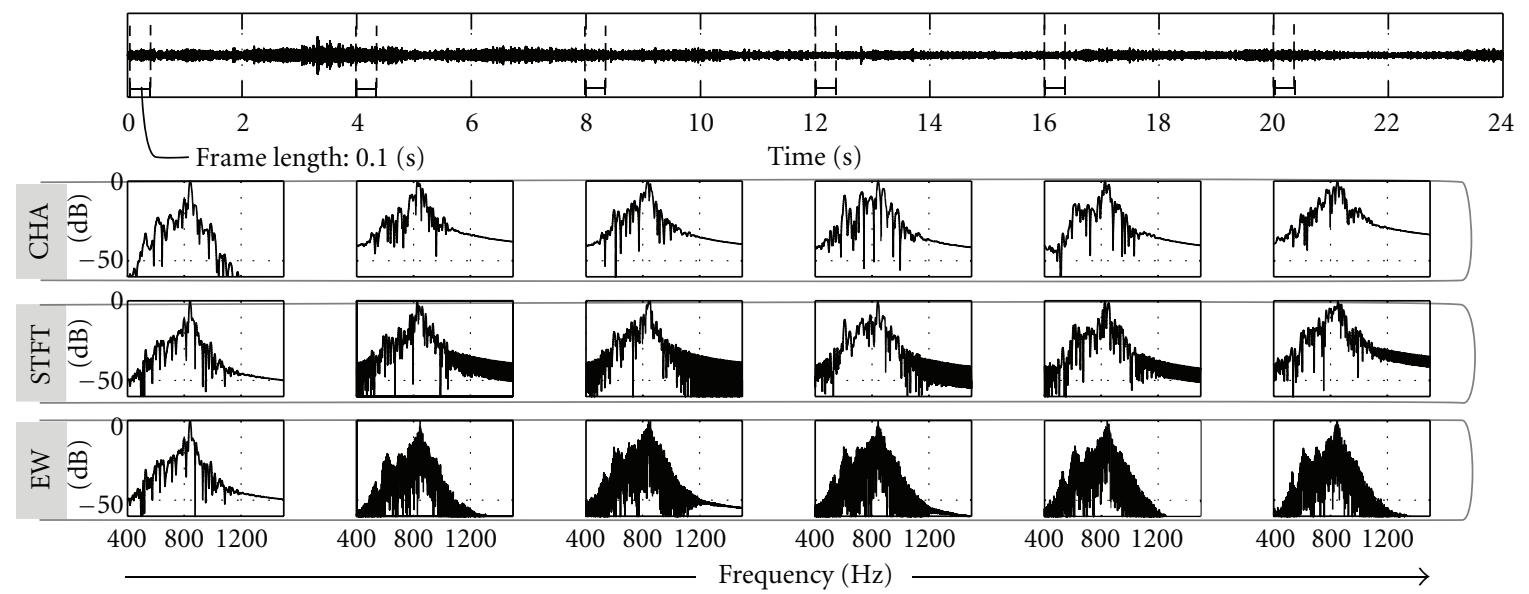

Figure 5: Examples of analysis.

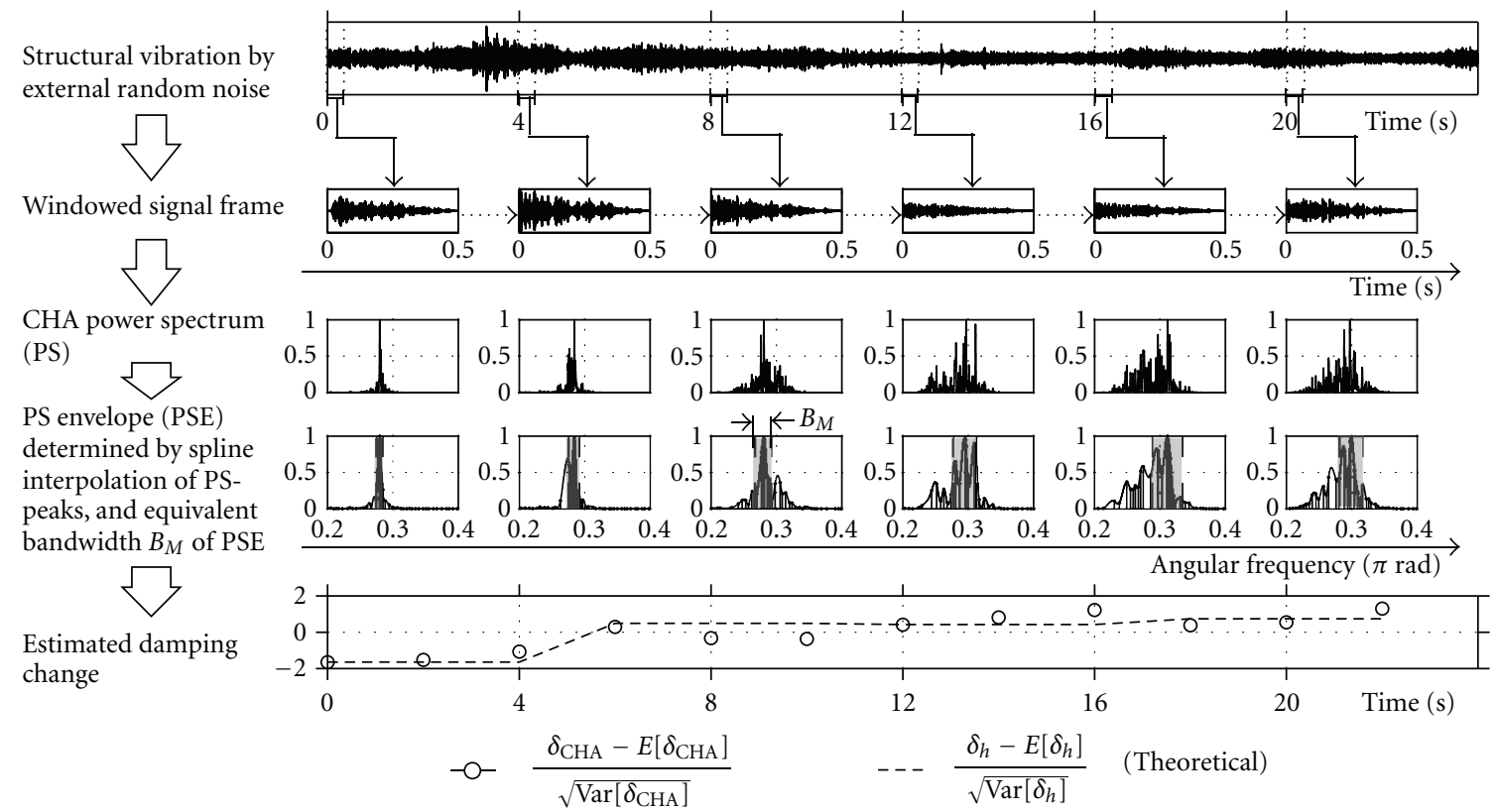

FIGURE 6: Monitoring examples of $\delta$ transition by CHA analysis.

Consequently, CHA can be formulated using triangular windowing,

$$
\begin{aligned}
\operatorname{CHA}\left(n, z^{-1}\right)= & \sum_{m=0}^{n} w(m) x(m) z^{-m} \\
= & \left\{x(0) z^{-0}\right\}+\left\{x(0) z^{-0}+x(1) z^{-1}\right\}+\cdots \\
& +\left\{x(0) z^{-0}+x(1) z^{-1}+\cdots+x(n) z^{-n}\right\} \\
= & n x(0) z^{-0}+(n-1) x(1) z^{-1}+\cdots+x(n) z^{-n},
\end{aligned}
$$

in the $z$-plane including the unit circle where the Fourier transform is defined as shown in Figure 4(b). Panels (c) and (d) illustrate other possible methods for comparison, that is, short-time Fourier transformation (STFT) and exponential windowing $(\mathrm{EW})$. The STFT of the input signal frame $x(n)$ is formulated as

$$
\operatorname{STFT}\left(n, z^{-1}\right)=\sum_{m=0}^{n} x(m) z^{-m}
$$

with rectangular windowing (nonspectral accumulation). The EW method is a general analytical method for diagnostics with exponential forgetting from the beginning of the observation. The EW method does not need frame processing and is formulated as

$$
\operatorname{EW}\left(n, z^{-1}\right)=\sum_{m=0}^{n} \mathrm{e}^{-\alpha m / f s} x(m) z^{-m},
$$

with all input signals $x(n)$ having a sampling frequency $f s$. 


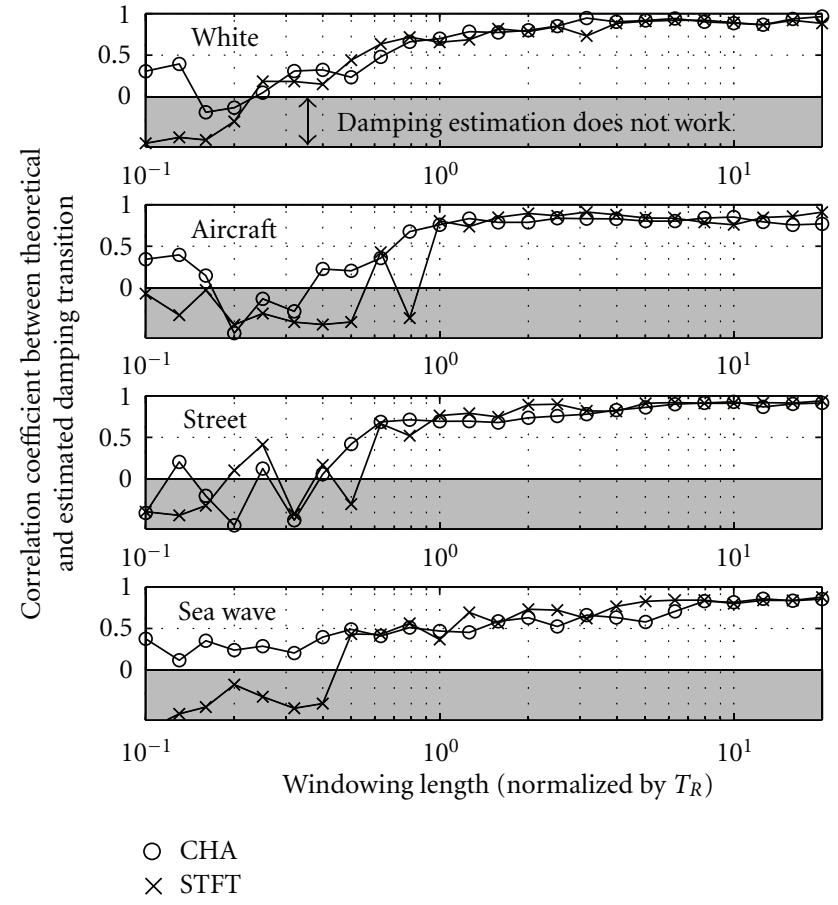

Figure 7: Correlation of damping constants estimated from the random vibration records and by using CHA and STFT on the impulse response data.

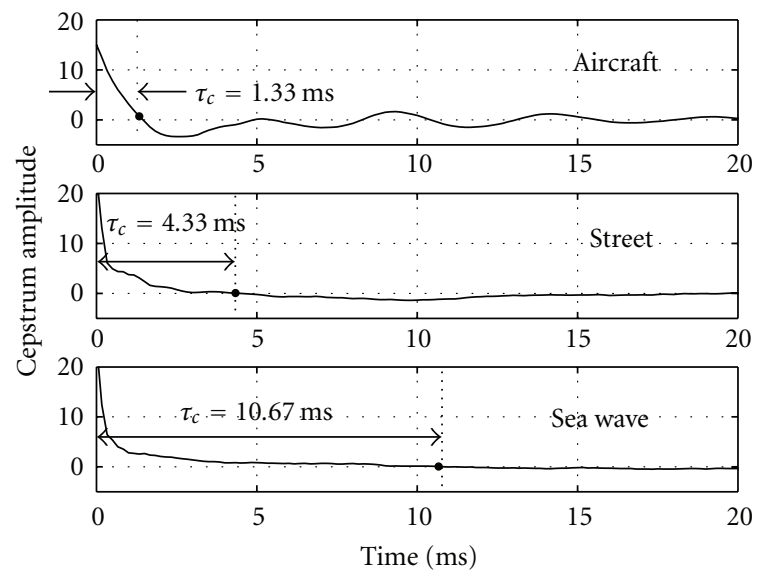

FIgURE 8: Amplitude cepstrum of external source signals.

Figure 5 shows examples of spectral analysis for each method. The CHA and STFT examples have a 0.1 (s) frame length, whereas the EW example uses all past signals and applies an exponentially decaying window function to all the data from the past that are to be used for the analysis at the present time. CHA also uses data from the past in the frame, but it has a triangular widowing function instead of an exponentially decaying one. Note that accumulation and restoration could also be used in CHA in order to adapt to the time varying behavior to be monitored and diagnosed.

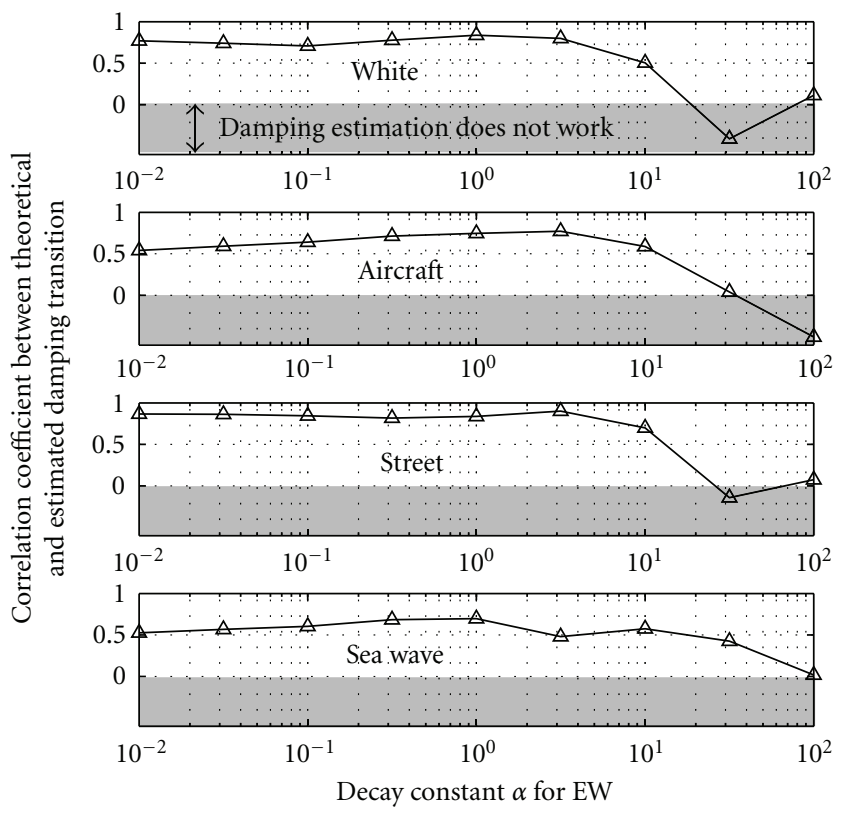

FIgure 9: Correlation of damping constants estimated from the random vibration records by using EW and from the impulse response data.

The modal bandwidth $B_{M}$ denotes the spectrum extent evaluated as an equivalent rectangular spectrum with the same area. The damping constant can be estimated by

$$
\delta=\frac{B_{M}}{\pi} .
$$

However, there are a lot of peaks that the power spectrum of a windowed signal frame when a random vibration source is used. The $B_{M}$ is calculated from the power spectrum envelope. Figure 6 shows the example of calculating $B_{M}$ and monitoring the system's damping changes with the CHA method. The top panel shows the recorded structural vibration signal. This signal is framed and windowed in the next step. The framing point is selected every 2 seconds. However, due to space limitations, the spectrum in this figure is shown only every 4 seconds. The CHA power spectrum is calculated and the peaks selected. The power spectrum envelope is estimated by spline interpolation. The spectrum envelope is normalized by the maximum value. Finally, the damping changes are estimated from the changes in the modal bandwidth of the spectrum envelope.

\section{Estimation Results}

Figure 7 shows the correlation coefficient between the damping constants estimated from the random vibration records by CHA (or STFT) and the estimation from the impulse responses shown in Figure 2. The horizontal axis is the windowing length (normalized by the reverberation time 
$T_{R} \simeq 0.1(s)$ of $\left.h_{1}\right)$, and the vertical axes show the correlation coefficients. It can be seen that CHA "o" yields a better estimation than STFT " $\times$ " when the windowing length is short; in particular, the time variance of the source signal increases in the case of a sea wave. The time variance of the source signal is confirmed from the zero-cross period of the amplitude cepstrum $\tau_{c}$ shown in Figure 8.

Figure 9 shows the correlation coefficient between the damping constants estimated from the random vibration records by EW and the estimation from the impulse responses shown in Figure 2. The horizontal axis is the decay constant $\alpha$ of the exponential window $\mathrm{e}^{\alpha t}$. From this figure, one sees that the decay constant $\alpha$ does not depend on the source signal for stable estimation. In the conventional EW method, an effective constant $\alpha$ is chosen for the known source signal. However, if the characteristics of the transfer function and the source signal contained in the observation signal are both unknown, $\alpha$ cannot be adjusted automatically.

\section{Summary}

Numerical simulations and experiments using a scale model confirmed that CHA well-visualizes temporal changes in a damped vibrating system and modal overlap conditions from random vibration records corresponding to an unknown external noise source. This development opens up a potential new area in cumulative analysis by introducing a spectral accumulation function into the method of Berman and Fincham. Spectral properties of the transfer function can be emphasized and visualized by the CHA magnitudes even in a short interval of random vibration samples. Monitoring the damping constants estimated by CHA will make it possible to estimate or predict temporal changes in the system damping of a structure. The experimental study confirmed that CHA works better than exponential windowing analysis or a short-time Fourier transform when the windowing length is short. CHA thus seems to be a practical way of monitoring conditions and making system diagnostics without a specific external source.

\section{Acknowledgment}

This work was supported by a Grant from the Japan Society for the Promotion of Science, KAKENHI (21860084).

\section{References}

[1] R. H. Lyon, "Progressive phase trends in multi-degree-offreedom systems," Journal of the Acoustical Society of America, vol. 73, no. 4, pp. 1223-1228, 1983.

[2] P. J. Halliday and K. Grosh, "Maximum likelihood estimation of structural wave components from noisy data," Journal of the Acoustical Society of America, vol. 111, no. 4, pp. 1709-1717, 2002.

[3] J. G. McDaniel and W. S. Shepard, "Estimation of structural wave numbers from spatially sparse response measurements," Journal of the Acoustical Society of America, vol. 108, no. 4, pp. 1674-1682, 2000.
[4] Y. Hirata, "A method for monitoring invisible changes in a structure using its non-stationary vibration," Journal of Sound and Vibration, vol. 270, no. 4-5, pp. 1041-1044, 2004.

[5] Y. Hirata, M. Tohyama, M. Matsumoto, and S. Gotoh, "Health monitoring for a structure using its nonstationary vibration," Advances in Acoustics and Vibration, vol. 2010, Article ID 696512, 2010.

[6] Y. Takahashi, M. Tohyama, and Y. Yamasaki, "Cumulative spectral analysis for transient decaying signals in a transmission system including a feedback loop," Journal of the Audio Engineering Society, vol. 54, no. 7-8, pp. 620-629, 2006.

[7] Y. Takahashi, T. Taniguchi, and M. Tohyama, "Structural condition monitoring by cumulative harmonic analysis of random vibration," Advances in Acoustics and Vibration, vol. 2008, Article ID 261758, 2008. 

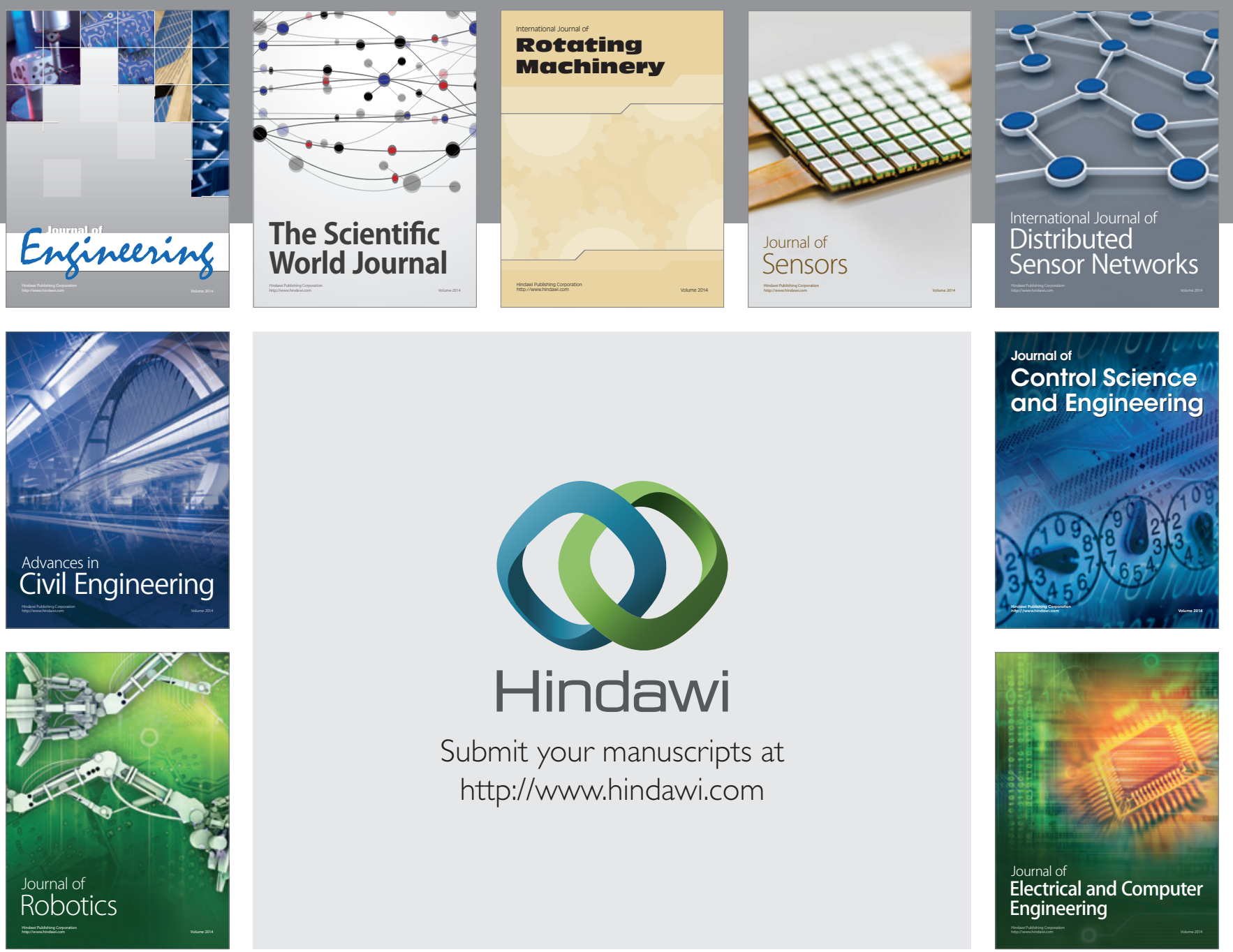

Submit your manuscripts at

http://www.hindawi.com
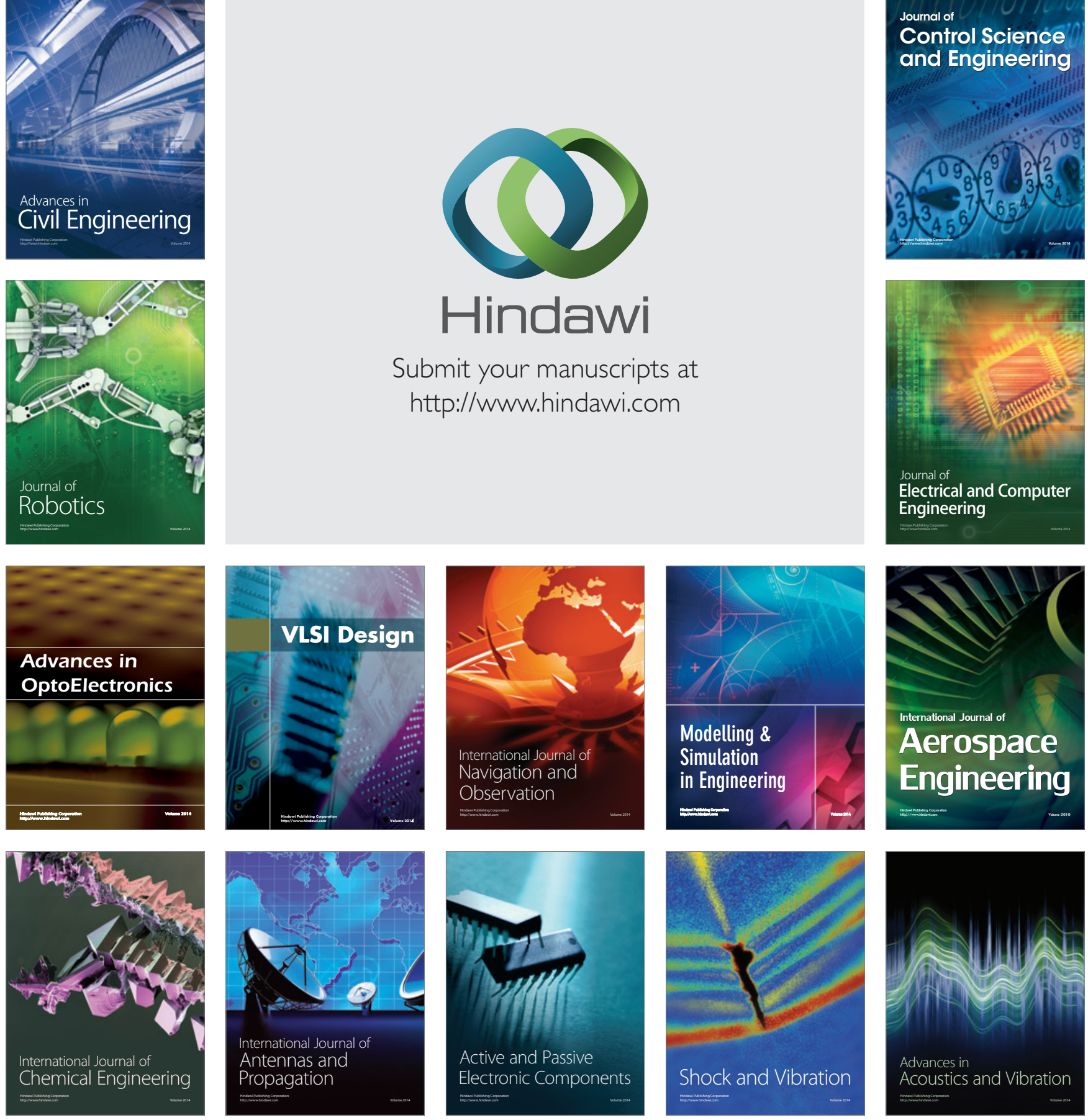